\title{
The relationship between pitch and space in congenital amusia
}

\author{
Victoria J. Williamson*, Gianna Cocchini, Lauren Stewart \\ Psychology Department, Goldsmiths, University of London, London SE14 6NW, UK
}

\section{A R T I C L E I N F O}

\section{Article history:}

Accepted 26 February 2011

Available online $\mathrm{xxxx}$

\section{Keywords:}

Congenital amusia

Visuo-spatial processing

Shepard-Metzler Mental Rotation

Corsi Blocks Task

Visual Patterns Test

\begin{abstract}
A B S T R A C T
Congenital amusia manifests as a lifelong difficulty in making sense of musical sound. The extent to which this disorder is accompanied by deficits in visuo-spatial processing is an important question, bearing on the issue of whether pitch processing draws on supramodal spatial representations. The present study assessed different aspects of visuo-spatial processing with a range of tasks (Shepard-Metzler Mental Rotation, Corsi Blocks Task, Visual Patterns Test) in 14 amusics and matched controls. The absence of a group difference on any of these tasks fails to support a previous claim that the disorder is strongly related to deficits in spatial processing. However, a subgroup of amusics, with significantly elevated thresholds on a pitch direction discrimination task relative to the rest of the group, were slower, but equally accurate, at Mental Rotation. This finding is discussed in relation to the nature of supramodal representations of contour and strategies for dynamic mental transformation.
\end{abstract}

(c) 2011 Elsevier Inc. All rights reserved.

\section{Introduction}

The ability to make sense of music is not restricted to those with musical training. Evidence of sophisticated musical processing is seen in early life (Hannon \& Trainor, 2007; Winkler, Haden, Ladinig, Sziller, \& Honing, 2009) and adults who lack musical training nevertheless display a high level of competence when tested for musical perception, memory and production abilities (Berkowska \& Dalla Bella, 2009; Bigand \& Poulin-Charronnat, 2006; Honing \& Ladinig, 2009; Levitin, 1994; Marmel \& Tillmann, 2009; Tillmann, Janata, Birk, \& Bharucha, 2008; Wise \& Sloboda, 2008).

An exception to this is the case of individuals with congenital amusia (hereafter 'amusia'), who do not acquire basic music perception and production abilities, despite normal exposure to music during development, normal peripheral hearing and typical performance on standardized tests of general cognitive abilities (Ayotte, Peretz, \& Hyde, 2002; Peretz et al., 2002). The condition may be considered alongside other congenital, neurodevelopmental disorders such as prosopagnosia, dyslexia, and dyscalculia (Tillmann et al., 2010).

Individuals with amusia are typically diagnosed using the Montreal Battery for the Evaluation of Amusia (MBEA), a battery of tests requiring discrimination of two short melodies that can differ on various musical dimensions (e.g. contour, rhythm and pitch intervals) as well as an assessment of meter perception and incidental memory (Peretz, Champod, \& Hyde, 2003). Individuals defined as amusic, according to the MBEA, exhibit primary difficulties in pitch perception: they have higher thresholds on tasks requiring the

\footnotetext{
* Corresponding author.

E-mail address: vickywilliamson01@gmail.com (V.J. Williamson).
}

detection of a pitch change (Foxton, Dean, Gee, Peretz, \& Griffiths, 2004; Hyde \& Peretz, 2004) and the discrimination of pitch direction (Foxton et al., 2004; Liu, Patel, Fourcin, \& Stewart, 2010; Williamson, McDonald, Deutsch, Griffiths, \& Stewart, 2010). In addition, they have difficulties maintaining single pitches in short-term memory (Gosselin, Jolicoeur, \& Peretz, 2009; Williamson et al., 2010), in memorizing pitch sequences (Tillmann, Schulze, \& Foxton, 2009; Williamson \& Stewart, 2010), in perceiving small changes in speech intonation (Liu et al., 2010; Patel, Wong, Foxton, Lochy, \& Peretz, 2008), and in vocal pitch performance (Dalla Bella, Giguère, \& Peretz, 2009; Hutchins, Zarate, Zatorre, \& Peretz, 2010).

While many studies of congenital amusia have concentrated on examining deficits in the processing of musical, chiefly pitchbased, stimuli, the disorder also affords an opportunity to test hypotheses concerning the type of representations that are utilized during musical processing - in particular, the extent to which musical processing involves visuo-spatial representations. The idea that we represent pitch visuo-spatially has face validity: pitch is arranged visuo-spatially on the musical stave in western music notation, and height (e.g. lower or higher) is the most commonly used linguistic metaphor for movement in pitch (Melara \& Marks, 1990; Mudd, 1963; Patel, 2008). Furthermore, two groups have independently demonstrated a spatial musical/pitch association of response codes (SMARC)/(SPARC) (Lidji, Kolinsky, Lochy, \& Morais, 2007; Rusconi, Kwan, Giordano, Umilta, \& Butterworth, 2006). This effect is demonstrated using a task in which participants have to classify tones according to their timbre (e.g. violin or piano) where the tones may be high or low in pitch. Participants make speeded classifications of the timbre of each tone, ignoring pitch, using a response that is vertically aligned. When responses 
are interrogated post hoc, there is a clear facilitation when highpitched tones are associated with a response that is high in vertical space, and low-pitched tones are associated with a response that is low in vertical space, compared with the reverse mapping. Such a finding suggests that the representation of pitch may be intrinsically spatial in nature.

Individuals with amusia provide a test of the extent to which musical and visuo-spatial stimuli share a common representational framework. If musical and visuo-spatial processing draw on partly shared representations or processes, then the pitch processing impairments seen in amusia may be expected to be accompanied by deficits in visuo-spatial processing. Some evidence supporting a common link between pitch and visuo-spatial processing in amusia was reported by Douglas and Bilkey (2007). These authors tested a group of amusics' using the classic Shepard-Metzler Mental Rotation paradigm (1971), a complex visuo-spatial cognition task that requires participants to carry out imagined mental transformations of 3D figures and which necessitates skills in object orientation discrimination, visual representation and dynamic spatial transformations (Podzebenko, Egan, \& Watson, 2002). Amusics made significantly more errors than matched controls during the Mental Rotation task and were also less influenced by pitch interference. Tillmann et al. (2010) failed to replicate this result and also reported no deficits in visuo-spatial attention in a separate cohort of amusics; however, this single negative result is not sufficient cause to reject the hypothesis of a relationship existing between pitch and visuospatial processing in amusia.

The present study was designed to further examine the visuospatial processing capacities of a group of amusic individuals, using a variety of visuo-spatial tests in order to determine which, if any, aspect(s) of visuo-spatial processing are deficient in amusia. Mental Rotation, as measured by the Shepard-Metzler task, encompasses object orientation discrimination, dynamic spatial transformations and sequential memory for spatial locations, and low performance on this test could imply a deficit at any, or all of these levels. Thus, we additionally tested memory for a sequence of spatial locations via the Corsi Blocks Task (hereafter, 'Corsi') (Milner, 1971) and memory for a two dimensional (2D) visual array, via the Visual Patterns Test (hereafter, 'VPT') (Della Sala, Gray, Baddeley, \& Wilson, 1997). Impaired performance on one or other of these two tasks, either alone or in combination with deficits in Mental Rotation ability, would allow us to better characterize the nature of any potential spatial processing deficit in amusia. In addition, in order to seek potential correlations between visuo-spatial measures and more fine-grained measures of pitch discrimination, we included two threshold-based measures of pitch perception: pitch detection and pitch direction discrimination (Liu et al., 2010; Williamson \& Stewart, 2010; Williamson et al., 2010).

\section{Method}

\subsection{Participants}

Participants for this study were screened using an online version of the scale subtest of the MBEA (Peretz et al., 2003). Participants who scored in the range of two standard deviations below the norm (i.e. 22/30 or less) on two occasions were invited to the laboratory to complete the scale, contour, pitch interval and rhythm subtests of the MBEA under controlled conditions. We calculated a pitch composite score for each individual and applied a criterion cut-off score of 65 for confirmation of amusia (Liu et al., 2010; Williamson \& Stewart, 2010; Williamson et al., 2010). Twenty-eight participants (14 individuals with amusia and 14 controls) took part in all tasks in the present experiment, in return for a small honorarium. The two groups were matched on gender, age, score on the National Adult
Reading Test (NART), Digit Span (Wechsler Adult Intelligence Scale; WAIS), number of years of formal education, and number of years of musical education (see Table 1 ).

\subsection{Psychophysical measures of pitch perception}

In advance of the visuo-spatial tasks, thresholds for the detection of a pitch change ('Pitch Detection' task) and discrimination of pitch direction ('Pitch Direction' task) were measured in all participants.

\subsubsection{Design}

Both tasks utilized a two-alternative forced choice AXB procedure where a single trial consisted of the presentation of three pure tones that were $600 \mathrm{~ms}$ in duration. For the Pitch Detection task, the target was a pitch glide, centered on $500 \mathrm{~Hz}$, while non-targets were steady-state tones of $500 \mathrm{~Hz}$. For the Pitch Direction task, targets and non-targets were pitch glides, centered on $500 \mathrm{~Hz}$. The target in this case was the glide with an excursion that was opposite in direction to the non-target glides. In both tasks, targets were upward or downward glides, with equal probability and appeared in the first or last position, at random. Participants were required to identify the position of the target by saying "first" or "last".

A 'two-up, one-down' staircase procedure with a variable step size was used to determine thresholds. The target was initially set to an excursion of 6 semitones. Two consecutive correct responses were required for a reduction in pitch excursion of the target while a single incorrect response resulted in an increase in pitch excursion. The latter was termed a 'reversal'. Reversals 1-4 employed a stepsize of 1 semitone; reversals $5-8$, a step-size of 0.1 semitones and reversals $9-14$, a step-size of 0.02 semitones. The protocol terminated after 14 reversals and a threshold was calculated based on the average pitch excursion of the target for the last six reversals.

\subsection{Visuo-spatial tasks}

\subsubsection{Mental Rotation}

2.3.1.1. Design. The stimuli were obtained from a library of 3D jpeg images created using the original Shepard-Metzler figures (Peters \& Battista, 2008). The library comprises 16 figures composed using 10 cubes of alternating white and grey cube surfaces that are presented on a white background. Each figure is rendered in $5^{\circ}$ steps of rotation from $0^{\circ}$ to $360^{\circ}$ in both the ' $x$ ' (the horizontal or 'abscissa') and ' $z$ ' (the vertical or 'ordinate') planes, for the original and the mirror reversed images. The present experiment comprised 32 trials where two jpeg images were presented side-by-side on screen; on half of these trials the two images were identical (hereafter 'same' trials; see Appendix A Fig. 1A and B) and on the other 16 trials the two images were mirror images (hereafter 'different' trials; see Appendix A Fig. 1C). Both same and different trials used the original 16 figures selected at random without replacement; four images that were rotated by $0^{\circ}$, four rotated by $60^{\circ}$, four by $120^{\circ}$, and four rotated by $180^{\circ}$. Within each of the four images per rotation degree, two were randomly assigned to be rotations in the ' $x$ ' plane and two in the ' $z$ ' plane.

Pilot testing indicated that some participants were able to identify different trials by selectively looking for mirror images. To prevent this strategy, 5 lure trials were incorporated into the design, whereby the two images shown had minor differences in the configuration of the blocks (see Appendix A Fig. 1) making a total of 37 experimental trials. Lure trials were not considered in the final analysis. Four additional trials (one at each degree of rotation; two in $x$ and two in $z$ ) were created to serve as practice trials.

2.3.1.2. Procedure. The procedure ran on Eprime software. Participants were instructed to observe the two images on the screen 
Table 1

Background participant details.

\begin{tabular}{|c|c|c|c|c|c|c|c|c|c|c|c|}
\hline Group & $N$ & & Age & NART & $\begin{array}{l}\text { Digit } \\
\text { Span }\end{array}$ & $\begin{array}{l}\text { Formal education } \\
\text { (years) }\end{array}$ & $\begin{array}{l}\text { Musical training } \\
\text { (years) }\end{array}$ & $\begin{array}{l}\text { MBEA } \\
\text { scale }\end{array}$ & $\begin{array}{l}\text { MBEA } \\
\text { contour }\end{array}$ & $\begin{array}{l}\text { MBEA } \\
\text { interval }\end{array}$ & $\begin{array}{l}\text { Pitch } \\
\text { composite }\end{array}$ \\
\hline \multirow[t]{2}{*}{ Amusic } & 14 & $\mu$ & 51.79 & 43.07 & 21.86 & 15.93 & 1.29 & 19.07 & 19.14 & 18.00 & 56.21 \\
\hline & & $\sigma$ & 11.38 & 4.36 & 3.23 & 2.06 & 3.02 & 2.56 & 2.66 & 2.18 & 6.38 \\
\hline \multirow[t]{4}{*}{ Control } & 14 & $\mu$ & 49.07 & 43.97 & 21.50 & 16.36 & .88 & 27.50 & 27.71 & 27.57 & 82.78 \\
\hline & & $\sigma$ & 10.67 & 3.46 & 2.85 & 2.82 & 1.55 & 2.21 & 2.23 & 2.71 & 6.00 \\
\hline & & $t$ & -.65 & .61 & -.31 & -.45 & -1.48 & 9.33 & 9.24 & 10.29 & 11.35 \\
\hline & & $p$ & .52 & .55 & .76 & .66 & .15 & $<.001$ & $<.001$ & $<.001$ & $<.001$ \\
\hline
\end{tabular}

and to indicate whether they were the same or different by pressing keys labeled ' $S$ ' or ' $D$ ' with their two index fingers. Images were defined as 'different' if no amount of rotation could bring them into alignment. On each trial participants first saw a central fixation cross for $3000 \mathrm{~ms}$ and were then presented with the two images. After making their response they triggered the next trial on their own time by pressing the spacebar. Participants completed four practice trials and were given visual feedback on their accuracy and their response time. Once each participant had correctly completed all the practice trials they proceeded to the 37 trials in the main task (including 5 lures). The order of trials was random for each participant and no feedback concerning accuracy or response time was given. The whole task took around $20 \mathrm{~min}$ to complete.

All the trials were scored using signal detection analysis. Responses to 'different' trials were categorized as 'hits' when participants responded 'different' and responses to 'same' trials were categorized as 'false alarms' when they responded 'different'. Accuracy was defined as the number of hits minus false alarms converted into a percentage, hereafter referred to as the guesscorrected data. The data were also scored using d prime analysis ( $Z$ score hits minus $Z$ score false alarms) but there were no significant differences in the pattern of results, so only guess-corrected data are reported in the results.

\subsubsection{Corsi Blocks Task}

2.3.2.1. Design. Nine irregularly positioned wooden cubes of $4 \mathrm{~cm}^{3}$ were arranged at fixed points on a flat wooden board $(26 \times 32 \mathrm{~cm})$ in order to create a standard Corsi board. The board was painted white and the blocks were labeled 1-9 in the standard arrangement with marks only viewable to the experimenter.

2.3.2.2. Procedure. The procedure was adopted from Orsini et al. (1987). During the task the experimenter and participant sat facing each other with the Corsi board in the center of the table. The experimenter then tapped on a number of these blocks with their index finger at a rate of one tap every $2 \mathrm{~s}$, moving directly from block to block. Once the experimenter withdrew their hand from the board, the participant attempted to tap the blocks in the same order. The procedure began with two practice trials at sequence length 2 and then two at length 3. Following correct execution of the practice trials, a maximum of five trials were then conducted at sequence length 3 . Participants were given feedback on their performance at the end of each trial. If three out of five trials at sequence length 3 were performed correctly then the number of blocks to be recalled on the next trial increased by one. The test stopped, either when the participant correctly recalled fewer than three sequences at a given sequence length or when three sequences at length 9 were correctly reproduced. The whole task took around $15 \mathrm{~min}$ to administer. Corsi span score was defined as the longest sequence length at which participants successfully recalled three sequences. This span score was then adjusted depending on participant age, sex and number of years of education (Orsini et al., 1987).

\subsubsection{Visual Patterns Test (VPT)}

2.3.3.1. Design. The patterns from Version A of the standardized VPT were used. The VPT materials consist of 39 images of a black and white matrix printed on card, with a set number of squares filled in black (corresponding to the sequence length; from 3 to 15). The size of the matrices increases by two cells for each increase in sequence length, and grid size accordingly expands from a minimum size of $2 \times 3$ to a maximum size of $5 \times 6$. For the present experiment the stimulus cards were scanned into jpeg images and presented on a blank white screen using an Eprime program. ${ }^{1}$ Recall of the patterns took place using blank paper templates.

2.3.3.2. Procedure. Participants were first read the standardized instructions from the VPT. Three practice trials using the same visual pattern (sequence length 4) were then used to demonstrate the task to participants. On each trial the participant was first provided with an answer template which corresponded exactly to the dimensions of the to-be remembered pattern stimulus. The participant then triggered presentation of the to-be remembered visual pattern by pressing the space bar. The pattern was displayed on screen for $3 \mathrm{~s}$ before it was replaced with a blank white screen. At this point the participant attempted to recall the on-screen pattern by placing crosses in their answer template to represent the squares that they recalled as being black. There was no time limit placed on responding and participants could correct a response if they wished. The experimenter provided feedback on each trial and a correct response was defined as an exact replication of the stimulus pattern. After completing the practice trials, participants were presented with three trials at each sequence length commencing at length 3 . If participants achieved one correct pattern replication from the three presented then sequence length was increased by one. Testing continued until the participant failed to correctly recall at least one of the patterns given at a particular sequence length. The entire procedure took around $15 \mathrm{~min}$ to complete.

\section{Results}

\subsection{Pitch thresholds}

Table 2 shows the average pitch thresholds for all 28 participants, expressed in semitones. The amusic and control groups did not differ significantly in their pitch detection thresholds (control $M=.14$ vs. amusic $M=.27$ ), but amusics had significantly higher thresholds for the Pitch Direction task (control $M=.17$ vs. amusic $M=.99$ ).

\subsection{Visuo-spatial tasks}

Individual and average group performance data for all three tasks (Mental Rotation, Corsi and VPT) is shown in Fig. 1. Initial

\footnotetext{
1 The scanned images were kindly provided by Louise Brown (Brown, Forbes, \&
} McConnell, 2006) 
Table 2

Average group threshold for Pitch Detection and Pitch Direction tasks expressed as proportion of a semitone.

\begin{tabular}{|c|c|c|c|c|}
\hline Group & $N$ & & Pitch detection threshold & Pitch direction threshold \\
\hline \multirow[t]{2}{*}{ Amusic } & 14 & $\mu$ & .27 & .99 \\
\hline & & $\sigma$ & .31 & 1.00 \\
\hline \multirow[t]{4}{*}{ Control } & 14 & $\mu$ & .14 & .17 \\
\hline & & $\sigma$ & .06 & .08 \\
\hline & & $U$ & -1.54 & -2.90 \\
\hline & & $p$ & .13 & .003 \\
\hline
\end{tabular}

(A) Mental Rotation

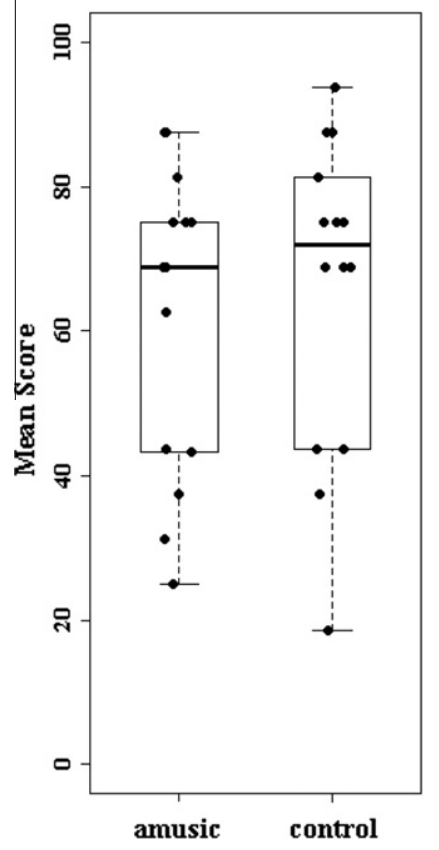

(B) Corsi Blocks

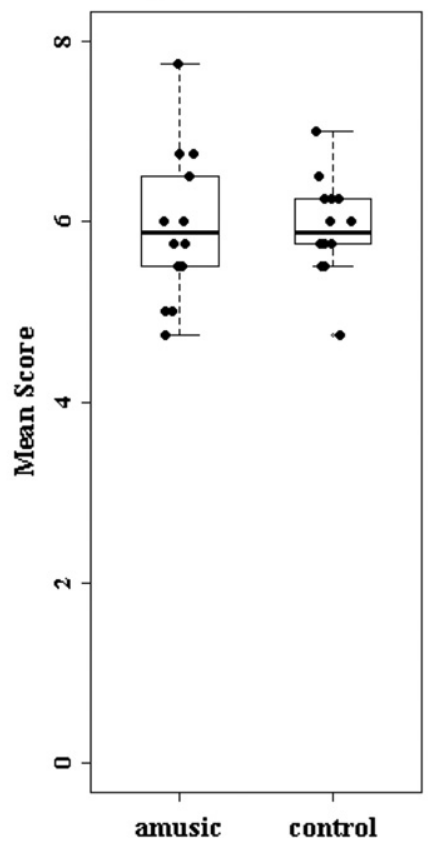

(C) Visual Patterns Test

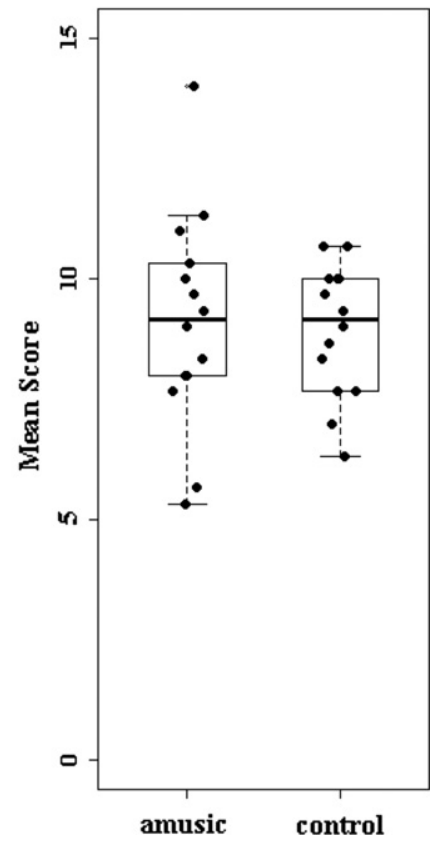

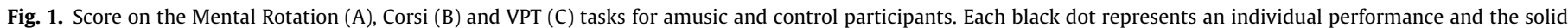
black bar marks the group median performance level.

analyses indicated that performance on the three tasks were not correlated (all $p>.10$ ) so the data were entered into a MANOVA with Group (control vs. amusic) as the between-subjects factor and performance on each of the three tasks as the dependent variable. No effects of Group were found for any of the tasks (all $p>.59$ ). This pattern of results remained when the upper amusic outlier was excluded from the VPT and the lower control outlier was removed from the Corsi.

\subsection{Mental Rotation: accuracy and response times}

Further analysis was carried out on the Accuracy (Fig. 2) and response time data ${ }^{2}$ (RT; Fig. 3) obtained from the Mental Rotation task.

Both measures were entered into ANOVAs with Group (control or amusic) as a between-subjects factor and Degree of Rotation $\left(0^{\circ}\right.$, $60^{\circ}, 120^{\circ}$, and $180^{\circ}$ ) as the within-subjects factor. No interaction was found between Group and Degree of Rotation for either Accuracy $(F(3,78)<1, p=.96)$ or $\mathrm{RT}(F(3,78)<1, p=.99)$, nor were there any effects of Group on either measure $(p>.59)$. Finally, the effect of Degree of Rotation was significant in both Accuracy

\footnotetext{
2 The data were analysed for RTs to all trials and RTs to correct trials only. There were no differences in the pattern of results that followed so only the latter are
} presented.

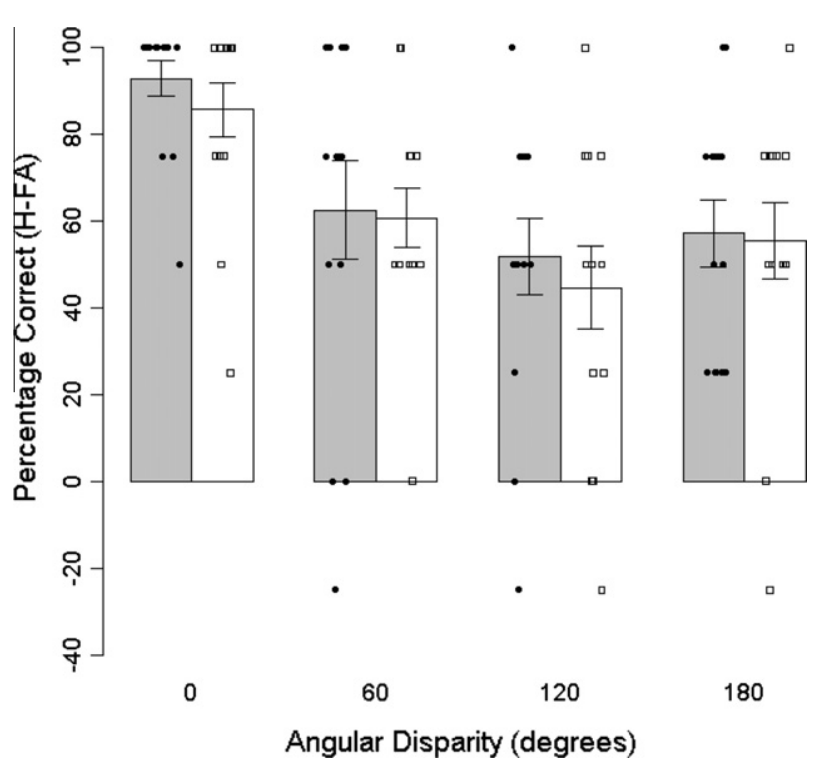

Fig. 2. Percentage correct of the guess corrected scores (H-FA) on the Mental Rotation task at all Degrees of Rotation $\left(0^{\circ}, 60^{\circ}, 120^{\circ}\right.$, and $\left.180^{\circ}\right)$ for amusics (white; squares) and controls (grey; circles). Error bars represent standard error. 


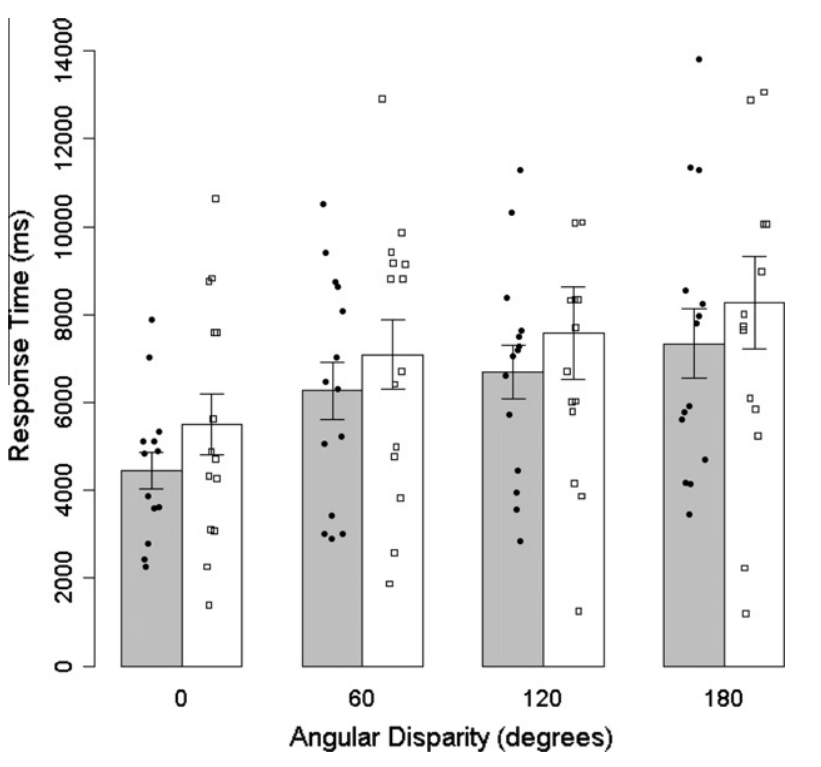

Fig. 3. Response times (ms) to correct trials only on the Mental Rotation task at all Degrees of Rotation $\left(0^{\circ}, 60^{\circ}, 120^{\circ}\right.$, and $\left.180^{\circ}\right)$ for amusics (white; squares) and controls (grey; circles). Error bars represent standard error.

$(F(3,78)=14.17, p<.001)$ and RT data $(F(3,78)=11.55, p<.001)$, reflecting the classic effect of an increase in rotation leading to longer response times and poorer accuracy in discriminating Shepard and Metzler figures.

\subsubsection{Correlations with Mental Rotation}

Both Douglas and Bilkey (2007) and Tillmann et al. (2010) reported a number of regressions and correlations between performances on the Mental Rotation task and other background measures including MBEA score, gender and score on pitch perception tasks. We have replicated these analyses and included new correlations on measures unique to the present experiment.

First, Douglas and Bilkey (2007) carried out a hierarchical regression on Mental Rotation scores, Gender and Group to determine the relationship between these factors and variance on MBEA contour subtest ${ }^{3}$. They report that Gender and Group accounted for $71 \%$ of the variance in MBEA contour subtest scores and that Mental Rotation score accounted for an additional $8 \%$. The same hierarchical regression carried out on the present data indicated that Gender and Group accounted for a similarly high proportion of the variance in MBEA contour score $(77 \%$; $p<.001)$, however, Mental Rotation accounted for only $.001 \%$ of the addition variance $(p=.74)$, a result which challenges evidence for a relationship between amusia (as defined by the contour subtest of the MBEA) and Mental Rotation ability. The same pattern of results emerged when using the pitch composite score from the MBEA.

Finally we analyzed the relationship between participants' psychophysical thresholds for pitch detection and pitch direction, and their Mental Rotation performance. We found no correlations between pitch detection thresholds and either measure of Mental Rotation performance ( $p s>.31)$. In addition we also found no correlation between the pitch direction discrimination threshold and Mental Rotation Accuracy $(p=.65)$. However, there was a significant correlation between pitch direction threshold and Mental Rotation RT $(r=.47, p=.01)$. Inspection of the data in Fig. 4 indicates that this effect is primarily driven by a subgroup of amusic

\footnotetext{
3 Douglas and Bilkey (2007) use this single subtest to define their groups as either amusic or control.
}

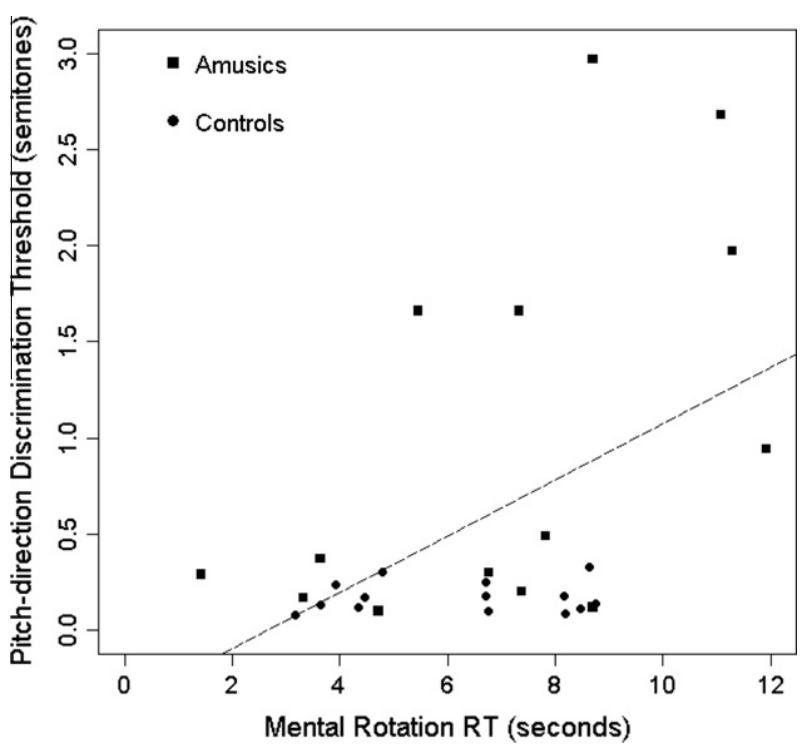

Fig. 4. Correlation plot showing the relationship between pitch direction discrimination thresholds (semitones) and Mental Rotation response time (RT; seconds) in both the control (circles) and amusic (squares) participants.

individuals whose thresholds for pitch direction discrimination are close to, or exceed, one semitone.

\subsubsection{Pitch direction discrimination thresholds and visuo-spatial performance}

It appears from the present data that individuals with higher (i.e. worse) thresholds for the discrimination of pitch direction are, to some degree, impaired (or at least slower) at Mental Rotation. We further investigated this issue by determining how this subgroup of amusics with particularly high pitch direction discrimination thresholds performed on this and all the other tests in the present experiment. The first step was to determine which amusics' thresholds differed significantly from the control group and to assess this we utilized Crawford and Garthwaite's (2002) t-test for individual scores. The thresholds of six amusics were found to be significantly different from those of the control group $(t>9.34, p<.001)$ suggesting that nearly half our amusic sample had higher (worse) thresholds for the discrimination of pitch direction. These six amusics were matched to a group of six control participants on all pre-test measures (see Table 3) and the MANOVA and ANOVAs were re-run to compare performance across these two groups for all visuo-spatial tasks.

As in the previous analysis of the larger sample there were no Group differences on the Corsi $(F<1, p=.69)$, VPT $(F=1.30$, $p=.28)$ or Mental Rotation tasks $(F<1, p=.71)$, and neither the Group effect nor the interaction between Group and Degree of Rotation were significant in the Mental Rotation ANOVA for Accuracy $(F<1, p=.53 ; F<1, p=.71)$. However, the Group effect was now significant in the Mental Rotation ANOVA for RT $(F=7.40$, $p=.02$ ) (no interaction with Degree of Rotation, $F=1.16, p=.34$ ). Due to the small number of observations in this sample we also analyzed the main group effects on mean task performance (collapsed across Degree of Rotation) using non-parametric Mann Whitney $U$ tests. Again there were no Group differences on the Corsi $(z=-.73, p=.49)$, VPT $(z=-1.04, p=.31)$ or Mental Rotation tasks $(z=-.41, p=.70)$, but there was a significant between-group difference in reaction time for the Mental Rotation task $(z=-2.56$, $p=.009$ ). This result, in combination with the correlation found between pitch direction threshold and Mental Rotation RT (Fig. 4), suggests that individuals with amusia who have significantly 
Table 3

Matching of the six high pitch direction threshold amusics to six controls on all pre-test measures.

\begin{tabular}{|c|c|c|c|c|c|c|c|c|c|c|c|}
\hline Group & $N$ & & Age & NART & $\begin{array}{l}\text { Digit } \\
\text { Span }\end{array}$ & $\begin{array}{l}\text { Formal education } \\
\text { (years) }\end{array}$ & $\begin{array}{l}\text { Musical training } \\
\text { (years) }\end{array}$ & $\begin{array}{l}\text { MBEA } \\
\text { scale }\end{array}$ & $\begin{array}{l}\text { MBEA } \\
\text { contour }\end{array}$ & $\begin{array}{l}\text { MBEA } \\
\text { interval }\end{array}$ & $\begin{array}{l}\text { Pitch } \\
\text { composite }\end{array}$ \\
\hline \multirow[t]{2}{*}{ Amusic } & 6 & $\mu$ & 58.83 & 43.00 & 21.17 & 15.50 & 2.00 & 20.17 & 19.50 & 18.50 & 58.17 \\
\hline & & $\sigma$ & 5.74 & 4.94 & 2.71 & 1.97 & 4.43 & 2.48 & 2.26 & 0.55 & 4.26 \\
\hline \multirow[t]{4}{*}{ Control } & 6 & $\mu$ & 54.67 & 45.17 & 21.00 & 16.50 & 1.33 & 26.50 & 27.67 & 27.33 & 80.83 \\
\hline & & $\sigma$ & 7.89 & 3.71 & 2.75 & 0.84 & 1.75 & 2.88 & 1.75 & 2.42 & 6.55 \\
\hline & & $t$ & -1.05 & .86 & -.08 & -1.14 & -.34 & 4.08 & 7.00 & 8.71 & 7.10 \\
\hline & & $p$ & .32 & .41 & .94 & .29 & .74 & .002 & $<.001$ & $<.001$ & $<.001$ \\
\hline
\end{tabular}

higher (worse) thresholds for the discrimination of pitch direction compared to controls are slower but no less accurate on the Mental Rotation task (controls $M=5.85 \mathrm{~s}(S D=1.74)$ vs. amusics $M=9.30 \mathrm{~s}$ $(S D=2.57))$.

\section{Discussion}

Previous studies have demonstrated that pitch can be mapped onto a vertical representation of space (Lidji et al., 2007; Rusconi et al., 2006) suggesting the existence of partly shared representations or processes for musical (e.g. pitch) and spatial processing. Individuals with congenital amusia provide a test of this association, since they are known to have difficulty in processing pitch. If pitch and space share a common representational dimension, then individuals with amusia may be expected to show deficits on tests of visuo-spatial processing.

A commonly used test of visuo-spatial processing is the Shepard-Metzler Mental Rotation task (1971), which requires participants to determine whether a rotated 2D schematic of a 3D object can be fitted into alignment with the $2 \mathrm{D}$ schematic of the unrotated object. This is a complex task that requires skills in object orientation discrimination, visual representation and dynamic spatial transformations (Podzebenko et al., 2002). Douglas and Bilkey (2007) reported that individuals with amusia were impaired at this task, though Tillmann et al. (2010) failed to replicate this finding. While the Mental Rotation task taps into multiple complex aspects of visuo-spatial processing, it by no means captures every facet. The present experiment, in addition to measuring Mental Rotation ability, also examined fundamental components of visuo-spatial processing: 2D visual pattern memory and serial recall of spatial sequential patterns, two abilities as yet unexplored in individuals with congenital amusia.

We found no difference between amusics' and controls' performance on a Mental Rotation task, as measured by both accuracy and response times, despite our successful replication of classic effects associated with angular disparity. We also showed that performance on the Mental Rotation task did not correlate with scores on the MBEA contour subtest, nor with MBEA pitch composite score or perceptual thresholds for the detection of a pitch change. Finally, we found no difference in group scores on either the Corsi (serial order recall of sequential spatial locations) or the VPT (2D visual pattern memory). In conclusion, in line with Tillmann et al. (2010) we found no correlation between Mental Rotation performance and standardized measures of congenital amusia and moreover, we also found a lack of association between amusia and two different measures of visuo-spatial memory: Corsi and VPT. Such convergent evidence, now replicated across two independent laboratories, casts doubt on the existence of a strong relationship between pitch and visuo-spatial processing in the majority of amusic individuals.

One finding from the present research, however, suggests that some individuals with amusia may indeed have subtle difficulties in visuo-spatial processing. Around half our amusic individuals had a significant impairment in the ability to discriminate the direction of a pitch change. The six amusics who showed significantly higher (worse) thresholds in pitch direction discrimination relative to the rest of the amusic group, responded significantly slower in the Mental Rotation task compared to a group of matched controls. It seems, therefore, that some individuals with amusia do encounter difficulty with Mental Rotation but that this impairment is limited to a slowing of response time and not overall accuracy. These individuals show no difference in performance on other visuo-spatial tasks.

Taking a wider view, we now consider one possible explanation as to why amusic individuals with poor direction discrimination thresholds performed more slowly on the Mental Rotation task. The Mental Rotation paradigm can be solved using an analogue strategy or an analytic "piecemeal" strategy. In the former strategy, object integrity is maintained for the purpose of holistic mental transformation; in the latter, individual object segments are manipulated sequentially (Cooper \& Podgorny, 1976; Kosslyn, 1981). The ability to transform objects holistically is thought to be governed by the "structure-preserving constraints of the underlying perceptual apparatus” (Shepard \& Cooper, 1982, p. 4). It has been proposed that the holistic processes which are sensitive to structure-preserving constraints may underlie both auditory and visual judgments where the shared constraint is stimulus contour (Cupchik, Phillips, \& Hill, 2001). The slowed Mental Rotation response times of our subgroup of amusics may reflect a difficulty in employing such supramodal (i.e. visual-auditory) representations of stimulus contour (Aksentijevic, Elliott, \& Barber, 2001; Monje-Garcia \& Aksentijevic, 2008) and consequently, a disproportionate reliance on suboptimal analytic instead of analogue strategies. Difficulty processing supramodal representations of contour might also go towards explaining these individuals' poorer pitch direction discrimination thresholds if such tasks rely to any degree on the use of visuo-spatial representations.

Notwithstanding the observed association between pitch direction thresholds and Mental Rotation response time in a subgroup of individuals, we find no evidence to support to support the claim that 'amusia is strongly linked to a deficit in spatial representation or processing' (Douglas \& Bilkey, 2007, p. 919). This conclusion does not directly challenge the wider evidence for the existence of a link between music and spatial processing (Lidji et al., 2007; Mudd, 1963; Rusconi et al., 2006), but rather suggests that such an association is unlikely to underlie the majority of amusics' cognitive difficulties with music perception.

\section{Acknowledgments}

We would like to thank all our amusic and control participants for their continued generous support. In addition we thank Sukhbinder Kumar and Tim Griffiths for their assistance with task development, and Fang Liu and Guy Peryer for their assistance with data collection. The authors were supported by an ESRC First Grant (RES/061/25/0155) awarded to LS. 


\section{Appendix A. Supplementary material}

Supplementary data associated with this article can be found, in the online version, at doi:10.1016/j.bandc.2011.02.016.

\section{References}

Aksentijevic, A., Elliott, M. A., \& Barber, P. J. (2001). Dynamics of visual grouping: Similarities in the organisation of visual and auditory groups. Visual Cognition, $8(3 / 4 / 5), 349-358$.

Ayotte, J., Peretz, I., \& Hyde, K. (2002). Congenital Amusia: A group study of adults afflicted with a music-specific disorder. Brain: A Journal of Neurology, 125(2), 238-251.

Berkowska, M., \& Dalla Bella, S. (2009). Acquired and congenital disorders of sung performance: A review. Advances in Cognitive Psychology, 5, 69-83.

Bigand, E. \& Poulin-Charronnat, B. (2006). Are we "experienced listeners"? A review of the musical capacities that do not depend on formal musical training. Cognition, 100, 100-130.

Brown, L. A., Forbes, D., \& McConnell, J. (2006). Limiting the use of verbal coding in the Visual Patterns Test. The Quarterly Journal of Experimental Psychology, 59(7) 1169-1176.

Cooper, L. A., \& Podgorny, D. (1976). Mental transformations and visual comparison processes: Effects of complexity and similarity. Journal of Experimental Psychology: Human Perception and Performance, 2, 503-514.

Crawford, J. R., \& Garthwaite, P. H. (2002). Investigation of the single case in neuropsychology: Confidence limits on the abnormality of test scores and test score differences. Neuropsychologia, 40, 1196-1208.

Cupchik, G. C., Phillips, K., \& Hill, D. S. (2001). Shared processes in spatial rotation and musical permutation. Brain and Cognition, 46, 373-382.

Dalla Bella, S., Giguère, J.-F., \& Peretz, I. (2009). Singing in congenital amusia. Journal of the Acoustical Society of America, 126, 414-424.

Della Sala, S., Gray, C., Baddeley, A., \& Wilson, L. (1997). The Visual Patterns Test: A new test of short-term visual recall. Suffolk, England: Thames Valley Test Company.

Douglas, K. M., \& Bilkey, D. K. (2007). Amusia is associated with deficits in spatial processing. Nature Neuroscience, 10, 915-921.

Foxton, J. M., Dean, J. L., Gee, R., Peretz, I., \& Griffiths, T. D. (2004). Characterization of deficits in pitch perception underlying 'tone deafness'. Brain: A Journal of Neurology, 127(4), 801-810.

Gosselin, N., Jolicoeur, P., \& Peretz, I. (2009). Impaired memory for pitch in congenital amusia. The Neurosciences and Music III - Disorders and Plasticity: Annals of the New York Academy of Sciences, 1169, 270-272.

Hannon, E. E., \& Trainor, L. J. (2007). Music acquisition: Effects of enculturation and formal training on development. Trends in Cognitive Sciences, 11, 466-472.

Honing, H., \& Ladinig, O. (2009). Exposure influences timing judgments in music Journal of Experimental Psychology: Human Perception and Performance, 35 281-288.

Hutchins, S., Zarate, J. M., Zatorre, R., \& Peretz, I. (2010). An acoustical study of vocal pitch matching in congenital amusia. Journal of the Acoustical Society of America, 127(1), 504-512.

Hyde, K., \& Peretz, I. (2004). Brains that are out of tune but in time. Psychological Science, 15(5), 356-360.

Kosslyn, S. M. (1981). The medium and the message in mental imagery: A theory. Psychological Review, 88, 46-66.

Levitin, D. J. (1994). Absolute memory for musical pitch: Evidence from the production of learned melodies. Perception and Psychophysics, 56, 414-423.

Lidji, P., Kolinsky, R., Lochy, A., \& Morais, J. (2007). Spatial associations for musica stimuli: A piano in the head? Journal of Experimental Psychology: Human Perception and Performance, 33, 1189-1207.
Liu, F., Patel, A. D., Fourcin, A., \& Stewart, L. (2010). Intonation processing in congenital amusia: Discrimination, identification and imitation. Brain. doi:10.1093/brain/awq089.

Marmel, F., \& Tillmann, B. (2009). Tonal priming beyond tonics. Music Perception, 26(3), 211-221.

Melara, R. D., \& Marks, L. E. (1990). Processes underlying dimensional interactions: Correspondences between linguistic and nonlinguistic dimensions. Memory and Cognition, 18, 477-495.

Milner, B. (1971). Interhemispheric differences in the localization of psychological processes in man. British Medical Bulletin, 27, 272-277.

Monje-Garcia, L., \& Aksentijevic, A. (2008). The effects of melodic contour on Mental Rotation of visual patters: Cross-modality, structure and complexity. In Proceedings of the 8th international conference of cognitive science, Seoul: Korea.

Mudd, S. A. (1963). Spatial stereotypes of four dimensions of pure tone. Journal of Experimental Psychology, 66, 347-352.

Orsini, A., Grossi, D., Capitani, E., Laiacona, M., Papagno, C., \& Vallar, G. (1987). Verbal and spatial immediate memory span: Normative data from 1355 adults and 1112 children. The Italian Journal of Neurological Sciences, 8(6), 537-548.

Patel, A. D. (2008). Music, Language, and the Brain. New York: Oxford University Press.

Patel, A. D., Wong, M., Foxton, J., Lochy, A., \& Peretz, I. (2008). Speech intonation perception deficits in musical tone deafness (congenital amusia). Music Perception, 25, 357-368.

Peretz, I., Ayotte, J., Zatorre, R. J., Mehler, J., Ahad, P., Penhune, V. B., et al. (2002). Congenital amusia: A disorder of fine-grained pitch discrimination. Neuron, 33, 185-191.

Peretz, I., Champod, A.-S., \& Hyde, K. L. (2003). Varieties of musical disorders. The Montreal Battery of Evaluation of Amusia. Annals of the New York Academy of Sciences, 999, 58-75.

Peters, M., \& Battista, C. (2008). Applications of mental rotation figures of the Shepard and Metzler type and description of a mental rotation stimulus library. Brain and Cognition, 66(3), 260-264.

Podzebenko, K., Egan, G. F., \& Watson, J. D. G. (2002). Widespread dorsal stream activation during a parametric mental rotation task, revealed with functional magnetic resonance imaging. Neuroimage, 15, 547-558.

Rusconi, E., Kwan, B., Giordano, B. L., Umilta, C., \& Butterworth, B. (2006). Spatial representation of pitch height: The SMARC effect. Cognition, 99(2), 113-129.

Shepard, R. N., \& Cooper, L. (1982). Mental images and their transformations. Cambridge MA: MIT Press.

Shepard, R. N., \& Metzler, D. (1971). Mental rotation of three-dimensional objects. Science, 171, 701-703.

Tillmann, B., Janata, P., Birk, J., \& Bharucha, J. J. (2008). Tonal centers and expectancy: Facilitation or inhibition of chords at the top of the harmonic hierarchy? Journal of Experimental Psychology: Human Perception and Performance., 34(4), 1031-1043.

Tillmann, B., Jolicœur, P., Ishihara, M., Gosselin, N., Bertrand, O., et al. (2010). The amusic brain: Lost in music, but not in space. PLOS ONE, 5(4), e10173. doi:10.1371/journal.pone.0010173.

Tillmann, B., Schulze, K., \& Foxton, J. M. (2009). Congenital amusia: A short-term memory deficit for nonverbal, but not verbal sounds. Brain and Cognition, 71(3), 259-264.

Williamson, V. J., McDonald, C., Deutsch, D., Griffiths, T. D., \& Stewart, L. (2010). Faster decline of pitch memory over time in congenital amusia. Advances in Cognitive Psychology, 6, 15-22.

Williamson, V. J., \& Stewart, L. (2010). Memory for pitch in congenital amusia: Beyond a fine-grained pitch discrimination problem. Memory, 18(6), 657-669.

Winkler, I., Haden, G., Ladinig, O., Sziller, I., \& Honing, H. (2009). Newborn infants detect the beat in music. Proceedings of the National Academy of Sciences, 106(7), 2468-2471. doi:10.1073/pnas.0809035106.

Wise, K. J., \& Sloboda, J. A. (2008). Establishing an empirical profile of self-defined 'tone deafness': Perception, singing performance and self-assessment. Musicae Scientiae, 12(1), 3-26. 